\title{
News
}

Transfus Med Hemother 41 | 2 | 14

\section{Exjade $^{\circledR}$ (Deferasirox):}

\section{Eisen in Balance - Therapiemanagement bei MDS- und Thalassämie-Patienten}

Im Rahmen des 31. Deutschen Krebskongresses fand das Novartis Oncology Meet the Experts «Eisen in Balance: Therapiemanagement bei MDS- und Thalassämie-Patienten» statt. Referenten waren Prof. Dr. Wolf-Karsten Hofmann, Direktor der III. Medizinischen Klinik der Universitätsmedizin Mannheim, der zu Myelodysplastischen Syndromen (MDS) referierte, und Dr. Regine Grosse, Ärztin an der II. Medizinischen Klinik und der Kinder-Hämatologie und-Onkologie des Universitätsklinikums Hamburg-Eppendorf, die sich den Thalassämien widmete.

\section{Eisenüberladung bei MDS}

MDS sind erworbene Erkrankungen des Knochenmarks bzw. des Blutes, die durch eine ineffektive Blutbildung im Knochenmark gekennzeichnet sind [1]. Von den Reifungs- und Funktionsstörungen können eine oder mehrere Zelltypen im Blut betroffen sein [1]. MDS können zudem bei manchen Patienten zu einer akuten myeloischen Leukämie (AML) führen [1]. Ein großer Teil der MDS-Patienten entwickelt im Laufe der Erkrankung eine Anämie und ist dann auf eine regelmäßige Gabe von Erythrozytenkonzentrat(EK)Einheiten angewiesen. Täglich verliert der Körper 1-2 mg Eisen über Darm, Urin und Haut [2]. Eine EK-Einheit enthält jedoch $250 \mathrm{mg}$ Eisen, sodass es durch die kontinuierliche Zufuhr von Eisen über die Transfusionen bei chronisch transfundierten MDS-Patienten zu einer Eisenüberladung z.B. in Leber und im Herzen kommt [3]. Diese Anreicherung von Eisen ist mit Hilfe eines Eisenchelators wie Deferasirox behandelbar [1].

Von einer Therapie mit Deferasirox profitieren chronisch transfundierte MDS-Patienten in vielerlei Hinsicht: Die Hämatopoese kann sich verbessern [4-6] und der Transfusionsbedarf als Folge davon sinken [6]. Eine bei der jüngsten
Jahrestagung der American Society of Hematology (ASH) in New Orleans vorgestellte US-amerikanische Registerstudie zeigt außerdem, dass MDS-Patienten, die mindestens 6 Monate lang eine Eisenchelat-Therapie erhalten hatten, mehr als doppelt so lang lebten (median 102,5 vs. 48,7 Monate; $p<0,0001$ ) und später und seltener eine AML entwickelten (nach median 77 vs. 45,6 Monaten; $p<0,0001$ ) als Betroffene ohne Eisenchelation [7].

Deferasirox reduziert Eisenüberladung bei $\beta$-Thalassämie major

Die Thalassämien zählen zu den Hämoglobinopathien. Darunter versteht man eine Gruppe von angeborenen hämatologischen Erkrankungen, die auf eine Störung der Hämoglobin-Produktion, verursacht durch Mutationen in den Hämoglobin-Genen, zurückzuführen sind [8]. Abhängig davon, von welchem Thalassämie-Syndrom der Patient betroffen ist, kommt es zu einer mehr oder weniger ineffektiven Hämatopoese. Die $\beta$-Thalassämie major ist die in der Regel homozygote oder gemischt-heterozygote $\beta$-Thalassämie-Form [8]. Je stärker die Hämatopoese gestört ist, desto eher benötigen die Patienten regelmäßige Bluttransfusionen. Da es sich um eine Erbkrankheit handelt, die zurzeit noch nicht kurativ behandelt werden kann - außer in Einzelfällen mit Hilfe einer Stammzelltransplantation - muss die Transfusionsbehandlung bereits im ersten Lebensjahr beginnen und lebenslang erfolgen [8]. Auch hier kommt es aufgrund der chronischen Transfusionsabhängigkeit der Thalassämie-Patienten zur Entwicklung einer Eisenüberladung, die wiederum mithilfe einer Eisenchelat-Therapie mit Deferasirox behandelt werden kann. Erfolgt keine Eisenchelation, versterben die Betroffenen an den Folgen der Organschäden, die durch die
Eisenüberladung verursacht werden, meist bereits vor Erreichen des 30. Lebensjahres [9].

Die Therapie mit Deferasirox bietet auch den Thalassämie-Patienten Vorteile: Die Leber- und Herzeisenüberladung wird reduziert [10], eine Anreicherung von Eisen in den hormonbildenden Organen wird verhindert [11] - dies wirkt sich positiv auf das Überleben aus [12]. Die Lebensqualität kann sich durch die nur 1-mal tägliche orale Verabreichung verbessern. Im Vergleich zu der wesentlich weniger komfortablen subkutanen Dauerinfusion von Deferoxamin [13] zeigt Deferasirox [14] die gleiche Wirksamkeit, wie sich in der Verlängerung einer internationalen Phase-II-Vergleichsstudie zwischen Deferasirox und Deferoxamin zeigte, die ebenfalls beim ASH-Kongress vorgestellt wurde [10].

\section{Quelle}

Novartis Oncology Meet the Experts «Eisen in Balance: Therapiemanagement bei MDS- und Thalassämie-Patienten», im Rahmen des 31. Deutschen Krebskongresses, 20.02.2014 in Berlin

\section{Referenzen}

www.dgho-onkopedia.de/de/onkopedia/leitlinien/mds.

2 www.leukaemie-hilfe.de/download-informationen.html? \&no_cache $=1 \&$ tx_drblob_pil[downloadUid] $=76$.

3 Leitch HA et al.: Hematology. Am Soc Hematol Educ Program. 2009:664-672.

4 Pennell DJ et al.: ASH 2013; abstr \#1018.

5 Giagounidis A et al.: TumorDiagn \& Ther 2012;3:29-33.

6 Angelucci E et al.: Blood 2012;118, abstr \#425.

7 Lyons RM et al: ASH 2013; abstr \#2775.

8 www.awmf.org/uploads/tx_szleitlinien/025-017l_S1_ Thalassaemie.pdf.

9 Borgna-Pignatti C et al.: Haematologica 2004;89:1187-1193. 10 Pennell DJ et al.: ASH 2013; abstr \#1018.

11 Grosse R: Kinder- und Jugendmedizin 2013;13:A1-A8.

12 Brittenham GM: N Eng J Med 2011;364:146-156.

13 Desferal $^{\circledR}$ (Deferoxamin) Fachinformation (Stand: Mai 2011).

14 Exjade $^{\circledR}$ (Deferasirox) Fachinformation (Stand: Oktober 2013).

Weitere Informationen bei

Novartis Pharma GmbH

Ina Meyer

Roonstraße 25, 90429 Nürnberg

ina.meyer@novartis.com

\section{Ticker+++ Ticker+++ Ticker+++ Ticker+++ Ticker+++ Ticker+++ Ticker+++}

LEO Pharma. Einschränkungen der Nierenfunktion bei Patienten mit venösen Thromboembolien werden bei der initialen Antikoagulation häufig nicht ausreichend berücksichtigt. Dies zeigen die Ergebnisse einer aktuellen Versorgungsstudie, die im Rahmen eines Symposiums auf der 58. Jahrestagung der Gesellschaft für Thrombose- und Hämostaseforschung in Wien vorgestellt wurde. In diesem Zusammenhang wurde auch das Thrombosemanagement bei Tumorerkrankungen und bei Patienten in fortgeschrittenem Lebensalter diskutiert.

LEO Pharma GmbH

Thomas Schick

thomas.schick@leo-pharma.com
Hexal. 2014 laufen mehr biopharmazeutisch hergestellte Arzneimittel aus dem Patent als chemisch-synthetisierte Präparate. Damit steigen Stellenwert und Bedarf für qualitativ hochwertige Alternativen, also für Biosimilars. Unbestritten ist der Nutzen von Granulozyten-koloniestimulierenden Faktoren in der Prävention und Behandlung von Neutropenien bei Patienten, die eine myelotoxische Chemotherapie erhalten. Neu ist, dass auch hier Biosimilars eine führende Rolle einnehmen - so ist Filgrastim Hexal das in Deutschland am häufigsten verwendete kurzwirksame Filgrastim-Präparat.

HEXAL AG www.hexal.de
Daiichi Sankyo. Die direkten oralen Antikoagulanzien vereinfachen die orale Antikoagulation, denn die Therapie erfordert kein Monitoring der Blutgerinnung und ist dabei mindestens ebenso wirksam, aber sicherer als die Standardtherapie mit Vitamin-K-Antagonisten. Dies untermauern auch die Ergebnisse klinischer Phase-3-Studien wie ENGAGE AF-TIMI 48 und HOKUSAI-VTE, in denen der orale 1-mal täglich einzunehmende direkte Faktor-Xa-Inhibitor Edoxaban von Daiichi Sankyo untersucht wurde.

Daiichi Sankyo Deutschland $\mathrm{GmbH}$ Pia Nothing

pia.nothing@daiichi-sankyo.de 
Hämophilie und Schmerz - ein vielfach vernachlässigtes Problem

Viele Patienten mit Hämophilie leiden bereits ab dem frühen Kindesalter häufig unter Schmerzen, die die Lebensqualität wesentlich beeinträchtigen können. Diese Schmerzproblematik erfordert viel Aufmerksamkeit und spezielle Strategien im Umgang mit diesen Patienten, wie Hämophilie-Experten und Schmerztherapeuten auf einem von Novo Nordisk im Rahmen der Initiative Changing Possibilities in Haemophilia ${ }^{\circledR}$ veranstalteten Symposium berichteten.

\section{Schmerzen als ständige Begleiter}

Patienten mit Hämophilie kennen rezidivierende Schmerzen seit dem Kindesalter. Spätestens nach dem ersten Lebensjahr erhalten Patienten mit schwerer Hämophilie intravenöse (i.v.) Injektionen - bei regelmäßiger Prophylaxe mit Gerinnungsfaktorkonzentraten 1- bis 3-mal wöchentlich. Dazu kommen wiederholte Blutentnahmen. «Für die Kinder ist dies mit Schmerzen und Ängsten verbunden und bedeutet für alle Beteiligten eine besondere Herausforderung», erklärte Dr. Carmen Escuriola-Ettingshausen (Frankfurt/M.). In allen Altersgruppen sind akute Schmerzen bei Hämophilie ein Warnzeichen für Blutungen, in erster Linie in die Gelenke.

«Chronische Schmerzen entstehen meist aufgrund einer Arthropathie als Folge von Gelenkblutungen und stellen ein relevantes Problem dar», so Dr. Saskia Gottstein, Berlin. Rezidivierende Blutungen und durch sie verursachte Schmerzen führen zu einem Circulus vitiosus aus Schonhaltung, Muskelverspannung, Inaktivität, Muskelschwund und Fehlhaltungen. «Erschwerend kommt hinzu, dass Depressivität schon bei geringer Schmerzintensität $\mathrm{zu}$ Inaktivität führen kann», wie Prof. Boris Zernikow, Datteln, kommentierte.

\section{Besonderheiten der Schmerztherapie}

Die Hälfte der Patienten mit Hämophilie leidet an chronischen Gelenkschmerzen [1,2] Viele fühlen sich dadurch in Lebensqualität und Alltagsaktivität beeinträchtigt; bei einem Großteil wirkt sich dies auch auf die Stimmung aus [3, 4]. Laut Zernikow sind Schmerzverarbeitung und -bewältigung, schmerzbezogene Emotionen und Verhaltensmuster anders als bei chronischen Schmerzpatienten ohne Hämophilie. «Die Schmerzproblematik sollte möglichst frühzeitig in der Hämophilie-Therapie berücksichtigt und thematisiert werden», forderte Gottstein.

Empfehlungen zur Schmerztherapie geben die Leitlinien der World Federation of Hemophilia (WFH) [5]. «Die Gabe von Gerinnungsfaktoren zur Behandlung/Prophylaxe von Blutungen ist ein Teil der Schmerztherapie», so Gottstein. Ruhigstellung, Kühlung, Kompression sowie Physiotherapie und Analgetika lindern Schmerzen. Bei chronischen Gelenkschmerzen empfehlen die Experten die Mitbehandlung durch Psychotherapeuten/Psychologen und/oder Schmerztherapeuten. Entscheidend für eine bessere Versorgung von Schmerzpatienten ist ihrer Ansicht nach eine gute interdisziplinäre Zusammenarbeit.

«Physiotherapie ist häufig eine wirkungsvolle Schmerztherapie bei akuten und chronischen Beschwerden», betonte der Physiotherapeut Marc Rosenthal, Duisburg. «Herausragenden Stellenwert hat hier die individuelle Trainingstherapie», bestätigte Zernikow. Wichtig ist ein frühzeitiger Beginn, auch bei fehlenden oder wenig Schmerzen.
Behandlung von Kindern als besondere Herausforderung

Der Umgang mit Schmerzen bei Kindern, vor allem Kleinkindern mit Hämophilie ist nicht einfach. Die regelmäßigen Injektionen sind meist sehr belastend. Auch Eltern und medizinischem Personal wird viel Geduld abverlangt. «Bei der Bedarfstherapie wird die lebensnotwendige Faktorgabe oft verzögert, weil die Kinder aus Angst Traumata, Blutungen und Schmerzen zunächst verheimlichen», wie Escuriola-Ettingshausen berichtete. Aber es gibt Maßnahmen, die Injektionsschmerzen zu erleichtern: Ablenkung, das Kind aktiv in das Geschehen einbeziehen, Schaffen einer angenehmen Atmosphäre. Kinderärzte empfehlen ein topisches Lokalanästhetikum für die Punktionsstelle sowie Analgetika in kindgerechten Darreichungsformen (Sirup, Suppositorien).

\section{Quelle}

Satellitensymposium «Haemophilia and Pain - an Interdisciplinary Expert Panel Discussion» von Novo Nordisk, Jahrestagung der Gesellschaft für Thrombose- und Hämostaseforschung e.V. (GTH) am 14.02.2014 in Wien

\section{Referenzen}

1 van Genderen FR et al : Haemophilia 2006:12:147-153.

2 Riley RR et al.: Haemophilia 2011;17:839-845.

3 Wallny T et al.: Haemophilia 2001;7:453-458.

4 Poon JL et al.: Haemophilia 2012;18:699-707.

5 Srivastava A et al.: Haemophilia 2013;19:e1-e47.

Weitere Informationen bei

MED in MIND GmbH

Dr. Christiane Lentz

Dachauer Straße 38, 80335 München

Tel. +49 89 4520596-14, Fax: -29

dr.lentz@med-in-mind.de

\section{Ticker+++ Ticker+++ Ticker+++ Ticker+++ Ticker+++ Ticker+++ Ticker+++}

Celgene. Vorbehandelte Patienten mit einem Multiplen Myelom und einer ungünstigen Zytogenetik profitieren in vergleichbarer Weise von einer Therapie mit Pomalidomid $\left(\right.$ Imnovid $\left.^{\circledR}\right)$ wie Patienten mit einer Standardrisiko-Zytogenetik. Zudem verbessert Pomalidomid plus Dexamethason das Ansprechen und Überleben unabhängig von der Anzahl und Art der Vortherapien sowie vom Alter der Patienten. Dies zeigen mehrere Auswertungen der Phase-III-Studie MM-003, die beim ASH 2013 in New Orleans, USA, präsentiert wurden.

dna medical communications Deutschland

Sandra Wagner

swagner@dnamedcomms.com
QIAGEN N.V. plant die Markteinführung mehrerer neuartiger Produkte für den Bereich NextGeneration-Sequencing (NGS). Ihr Zweck ist es, die größten bestehenden Engpässe im NGSArbeitsablauf - die Probenvorbereitung und die Bioinformatik - zu beseitigen. Die neuen Produkte erweitern das rasch wachsende Portfolio an «universellen» Lösungen. Diese können sowohl mit jedem Sequenziergerät als auch mit QIAGENs GeneReader ${ }^{\mathrm{TM}}$-Plattform, die noch in diesem Jahr auf den Markt kommen soll, eingesetzt werden.

QIAGEN GmbH

Dr. Thomas Theuringer pr@qiagen.com
Bayer. Zur Optimierung der Therapie der superfizielle Venenthrombose (SVT) wird jetzt die randomisierte SURPRISE-Studie durchgeführt, in der Rivaroxaban und Fondaparinux bei 500 Patienten mit symptomatischer SVT und mindestens einem Risikofaktor für eine venöse Thromboembolie-Komplikation verglichen werden. Primäres Ziel der auf Nicht-Unterlegenheit angelegten Studie ist die Wirksamkeit und Sicherheit von Rivaroxaban (10 mg, oral 1-mal täglich) gegenüber Fondaparinux (2,5 mg, s.c. 1-mal täglich) zu evaluieren.

Bayer Vital GmbH

Jutta Schulze

jutta.schulze@bayer.com
KARGER

(ㄱ) 2014 S. Karger GmbH, Freibur

Fax + 497614520714 Information@Karger.com Information@Karg
www.karger.com 